\title{
Parietal EEG Theta/Beta Ratio as an Electrophysiological Marker for Extraversion-Related Differences
}

\author{
Daryna Ivaskevych ${ }^{1 *}$, Sergii Tukaiev ${ }^{1,2 *}$, Oleksandr Ivaskevych ${ }^{1}$, and Borys Palamar ${ }^{2,3}$ \\ ${ }^{1}$ National Taras Shevchenko University of Kyiv, Ukraine \\ ${ }^{2}$ National University of Physical Education and Sports of Ukraine, Scientific Research Institute, Ukraine
}

${ }^{3}$ Bogomolets National Medical University, Department of Social Medicine and Public Health, Kyiv, Ukraine

Submission: March 23, 2019; Published: April 23, 2019

*Corresponding author: Daryna Ivaskevych, National University of Physical Education and Sports of Ukraine, Scientific Research Institute, 1, Fizkultury St, 03150, Kyiv, Ukraine

Sergii Tukaiev, Taras Shevchenko National University of Kyiv, 64/13, Volodymyrs'ka St., 01601 Kyiv, Ukraine

\begin{abstract}
Personality traits describe the typical behavior of a person. These traits are related to cognitive processing. While extraversion represents a Behavior Activation System (BAS), neuroticism is a part of the Behavior Inhibition System (BIS). Independence of these systems allows assuming the existence of several personality types with different Extraversion and Neuroticism scores. These traits are also related to monoaminergic systems functioning, differences in EEG alpha asymmetry, and attention control during processing of emotionally laden stimuli. However, personality traits-related EEG differences are not consistent within studies. To investigate these relationships between EEG waves and personality, a study with 235 participants was conducted. After fulfilling the EPI questionnaire, resting-state EEG with closed eyes was recorded. Attention control and dominance of subcortical processes were evaluated with theta/beta ration (TBR) and the delta/beta ratio (DBR). Cluster analysis revealed the existence of two personality types with high Extraversion and low Neuroticism, and low Extraversion and high Neuroticism. These clusters had a small difference in TBR in the right parietal lobe. Spearman correlation supported this finding, revealed a connection of Extraversion with higher TBR in all areas except lateral prefrontal lobe and DBR in the parietal lobe, and left medial areas. Due to this, existence in attention control between introverts and extraverts is concluded.
\end{abstract}

Keywords: EEG Theta/Beta Ratio; Theta-Beta Ration; Extraversion; Neuroticism.

\section{Introduction}

Personality has a strong impact on behavior and cognitive functioning. Anxiety has an impact on cognitive capacity due to attentive bias and processing of unrelated to the task information, Eysenck, Derakshan, Santos \& Calvo (2007). Anxiety, which is related to personality traits, has a negative impact on inhibition function, processing efficiency, and shifting function [1]. In addition to the description of relationships between cognitive functions and personality trait, an association of personality traits with brain activity was investigated.

Personality differences are studied with different neuroimaging methods. EEG allows investigation of brain activity with high temporal resolution. Analysis of different frequency band activity provides and information about the interaction of cortex and subcortical structures in affective processes [2]. The dominance of slow waves over fast waves represents a reduced cortical control and motivational imbalance [3]. Slow wave to fast wave ratios allows investigation of attentional control and anxiety.
Neurobiology of traits revealed the hierarchy of personality traits and their relation to brain functioning. According to DeYoung [4], Neuroticism and Extraversion belong to different metatraits. While Extraversion belongs to Plasticity metatrait, which represents a tendency to explore, Neuroticism is a part of Stability metatrait, which is a tendency to restrain disruptive emotions. Several studies revealed Extraversion is a part of the Behavioral Approach System and connected to the dopaminergic system and endogenous opioid systems [5]. A positive association between Extraversion and brain activity during the resting state in nucleus accumbens, medial orbitofrontal cortex, amygdala, and striatum was found [6]. Similarly, an association of Neuroticism with brain activity in anterior cingulate cortex, insula, and amygdala was reported. This trait is related to chronical stress and reduced capability to mobilize [7]. Neuroticism is related to both Behavioral Inhibition System (BIS) and Fight-Flight-Freeze System (FFFS) which are modulated by serotonin and norepinephrine 
neurotransmitters. EEG and personality correlates were reported as well. Belonging of Extraversion and Neuroticism to different metatraits allows assumption of their independence and existence of several subjects' classes with various means.

Theta-beta ration (TBR) is related to both the performances of attention-demanding tasks and self-reported attentional control. TBR was inversely correlated with inhibitory control functioning measured and self-reported attention control [8]. This ratio is positively related to disadvantageous decision-making and ADHD [9]. Although these finding allowed hypothesizing that delta-beta ratio (DBR) is also related to attention control selfreport, Morillas-Romero et al. [10] revealed that anxiety-related decrease in attention control occurs only during performance of emotionally laden stimuli. While anxiety is related to Neuroticism [11], the difference between groups with a significant difference in this trait could result in TBR and DBR difference as well.

Knyazev et al. [12] reported a right temporoparietal junction (RTPJ) is involved in the processing of emotional social stimuli and the generation of a behavioral response. Mediation of these processes by RTPJ was positively associated with Agreeableness. In turn, Extraversion has a moderate correlation with the Hurst exponent in posterior sub-network of DMN (DMNp) [13]. Wacker [14] revealed the connection of extraversion with cognitive control during the AX continuous performance task and frontal EEG alpha asymmetry (FAA), which were changed to the opposite by the D2 blocker. In contrast, research of FAA in adolescence revealed no connection to Extraversion or other personality traits [15]. However, machine learning algorithms fail to predict personality differences, while open and closed eyes EEG were successfully distinguished [16]. The absence of consistency in these conclusions illustrates a necessity of further investigations of this field.

Rojas et al. [17] reported that parietal electrodes of the 10/20 International system of EEG electrodes placement are related to several Yeo networks. For P4 and P3 EEG seeds, the highest Sørensen-Dice similarity coefficient was obtained for the default mode network (DMN), frontoparietal (FP), and limbic networks. In the same study, a connection of P3 and P4 electrodes to ipsilateral lateral parietal cortex (LP), medial prefrontal cortex (MPF), middle temporal gyrus and ipsilateral inferior temporal cortex (IT) was revealed. Due to this, differences in these areas can be a representation of DMN, FP, and limbic networks. These findings make a link between EEG investigations and MRT studies what contributes to the analysis of gained results.

Due to described findings, this study is aimed to test the following hypotheses:

H1: several personality types with different Extraversion and Neuroticism scores exist.

H2: these personality types are different in physiological predictors of attentive control.

\section{Methods \\ Participants}

Participants were 235 students of Kyiv National Taras Shevchenko University, 62 of whom were female. All of them reported the absence of brain injuries or mental disorders. Participants' mean age is 18.45 with a standard deviation of 1.95 .

The study was approved by the Bioethics Commission of Educational and Scientific Centre "Institute of Biology and Medicine", Taras Shevchenko National University of Kyiv. Written informed consent was obtained from each subject in accordance with the World Medical Association (WMA) declaration of Helsinki (Helsinki, Finland, June 1964).Participants received additional points to their exam marks for participation in the study.

\section{Questionnaire}

Participants completed the adapted version of the Eysenck Personality Inventory (EPI). While the questionnaire was developed by Eysenck in 1963 [18], it was adapted by Shmelev A.G. in 1985. The Russian version of EPI includes 57 items. Extraversion and Neuroticism scales included 24 questions each. Nine items are aimed to evaluate sincerity (Lie Scale) [19]. Cronbach's alpha in the current sample was 0,710 for Extraversion and 0,753 for Neuroticism.

\section{Procedure}

Informed consent was obtained from participants. EPI was completed before EEG measurement of resting-state with closed eyes ( $3 \mathrm{~min}$ ). R Studio was used to analyze data.

\section{EEG Recordings}

Monopolar EEG record was performed with the use of EEG 23 Ch system Neurocom (Ukraine, XAI-MEDICA). Silver/silver chloride electrodes were symmetrically locates at anterior frontal (Fp1, Fp2), frontal (F3, F4, Fz, F7, F8), central (C3, C4, Cz) parietal (P3, P4, Pz), occipital $(01,02)$ and temporal (T3, T4, T5, T6) recording sites in accordance with international 10-20 scheme. The interconnected ear electrodes were used as a reference for all electrodes. The interelectrode impedance levels did not exceed $5 \mathrm{k} \Omega$. The sample rate of $500 \mathrm{~Hz}$ was used for all channels. We estimated the spectral power density (SPD) of all frequencies from 0.2 to $35 \mathrm{~Hz}$. Theta/beta ratio was calculated by dividing the theta power by the beta power, the delta/beta ratio by dividing the theta power by the delta power.

\section{Results}

\section{Summary}

Mean value of the Neuroticism scale was 12.88 with SD 4.31. Mean value of the Extraversion scale was 13.21 with SD 3.86. Theta/beta ratio (TBR) and delta/beta ratio (DBR) was calculated for participants who completed EPI. To evaluate variables distribution, the Shapiro-Wilk test was performed. For all variables, the test's significance was lower 0.01 for comparison with a normal distribution with the same mean and standard 
deviation. Due to this, the hypothesis of normality was rejected. Non-parametric tests were used for further analysis.

\section{Classification of Participants}

Table 1: Descriptive Statistics of Extraversion and Neuroticism for Groups.

\begin{tabular}{|c|c|c|c|c|}
\hline & \multicolumn{2}{|c|}{ Group 1 } & \multicolumn{2}{c|}{ Group 2 } \\
\hline & M & SD & M & SD \\
\hline Extraversion & 10.67 & 2.88 & 16.26 & 2.39 \\
\hline Neuroticism & 14.16 & 4.13 & 11.35 & 4.04 \\
\hline TBR (P4) & 3.54 & 3.47 & 4.75 & 4.75 \\
\hline
\end{tabular}

The PAM clustering algorithm was used to identify two clusters based on Extraversion and Neuroticism measures. The number of clusters was defined with the gap statistics method used for PAM cauterization. Gap statistic reached its maximum of 1.098 for two clusters. Due to this, participants were divided into two clusters with the PAM method. Gained cluster differed significantly in Extraversion and Neuroticism scales (Table 1). The first group was lower in Extraversion and higher in Neuroticism with significance $p<0.001$. Cohen's $d$ r equivalent of the difference between two means for Extraversion was 0.72, while the one for Neuroticism was -0.33 . Due to this, the effect size of the difference in Extraversion was bigger in comparison to the effect size of Neuroticism difference. There were no differences between groups in gender distribution. The group with high Neuroticism included 128 participants, while a group with high Extraversion included 108 participants. Both groups have different Extraversion and Neuroticism values from the ones in the sample.

The combination of high Extraversion and low Neuroticism without combinations without either simultaneously high or simultaneously low traits allows if these traits are opposites of one scale. If this assumption was true, Neuroticism and Extraversion be negatively correlated. To evaluate relationships between Extraversion and Neuroticism, the Spearmen correlation was performed. The correlation coefficient was low (rho $=.04$ ) and insignificant ( $\mathrm{p}$-value =.50). The hypothesis of relatedness of Neuroticism and Extraversion was rejected.

\section{Between-Group Differences in EEG}

No difference was found between groups in DBR. A significant difference was identified only for TBR in the P4 electrode. Cohen's $\mathrm{d}$ was 0.14 what can be recognized as a weak effect size. The first group's mean of TBR in P4 was 3.54 with SD 3.47, while the second's one was 4.76 with SD 4.75. To evaluate the relationships between personality traits and TBR in P4, a correlation analysis was conducted.

\section{Correlation Between Personality Scores and DBR}

Since the data were not normally distributed, Spearman correlation analysis was conducted to reveal relationships between Extraversion scales and selected slow wave - fast wave ratios. Low but significant correlation were revealed with DBR in Fp2 $(r=.158, p=.015)$, F3 ( $r=.167, p=.010)$, C3 ( $r=.132, p=.043)$,
C4 $(r=.162, p=.013), \mathrm{T} 6(r=.162, p=.013), P 3(r=.184, p=.005)$, $P 4(r=.198, p=.002), 01(r=.145, p=.027)$, and Pz $(r=.154$, $p=.018$ ) (Table 2).

Table 2: DBR Spearman Correlation with Extraversion.

\begin{tabular}{|c|c|c|}
\hline & $r$ & $p$ \\
\hline Fp2 & 0,158 & 0,015 \\
\hline F3 & 0,167 & 0,010 \\
\hline C3 & 0,132 & 0,043 \\
\hline C4 & 0,162 & 0,013 \\
\hline T6 & 0,162 & 0,013 \\
\hline P3 & 0,184 & 0,005 \\
\hline P4 & 0,198 & 0,002 \\
\hline 01 & 0,145 & 0,027 \\
\hline Pz & 0,154 & 0,018 \\
\hline
\end{tabular}

\section{Correlation Between Personality Scores and TBR}

More theta/beta rations correlated with Extraversion: Fp1 $(r=.218, p=.001), \mathrm{F} p 2(r=.222, p=.001), \mathrm{F} 3(r=.236, p=.000)$, T3 ( $r=.191, p=.003), \mathrm{T} 4(r=.207, p=.001), \mathrm{C} 3(r=.149, p=.022)$, C4 $(r=.217, p=.001), \mathrm{T} 5(r=.167, p=.010)$, T6 $(r=.192, p=.003)$, $P 3(r=.196, p=.003), P 4(r=.238, p=.000), 01(r=.209, p=.001)$, $\mathrm{O} 2(r=.168, p=.010), \mathrm{Fz}(r=.156, p=.017), \mathrm{Cz}(r=.180, p=.006)$, and $\mathrm{Pz}(r=.206, p=.001)$ (Table 3$)$. Correlations are stronger and more significant. Since correlation with $\mathrm{P} 4$ is the strongest one, between-group difference cannot be stated as non-existing.

Table 3: TBR Spearman Correlation with Extraversion.

\begin{tabular}{|c|c|c|}
\hline & $\boldsymbol{r}$ & $\boldsymbol{p}$ \\
\hline Fp1 & 0,218 & 0,001 \\
\hline Fp2 & 0,222 & 0,001 \\
\hline F3 & 0,236 & 0,000 \\
\hline T3 & 0,191 & 0,003 \\
\hline T4 & 0,207 & 0,001 \\
\hline C3 & 0,149 & 0,022 \\
\hline C4 & 0,217 & 0,001 \\
\hline T5 & 0,167 & 0,010 \\
\hline T6 & 0,192 & 0,003 \\
\hline P3 & 0,196 & 0,003 \\
\hline P4 & 0,238 & 0,000 \\
\hline 01 & 0,209 & 0,001 \\
\hline 02 & 0,168 & 0,010 \\
\hline Fz & 0,156 & 0,017 \\
\hline Cz & 0,180 & 0,006 \\
\hline Pz & 0,206 & 0,001 \\
\hline & & \\
\hline
\end{tabular}

\section{Discussion}

The first hypothesis was accepted after data analysis. The study revealed that two classes could be distinguished in the general population based on Extraversion and Neuroticism 
trains. One of these classes has a high Extraversion and low Neuroticism, another one has opposite scoring. Since Neuroticism and Extraversion are not related, there are at least two types of personality: the first with high Extraversion and low Neuroticism, the second with low Extraversion and High neuroticism. Since data were not normally distributed, the results of the cluster analysis are doubtful and require further examination.

Analysis results require rejection of the second hypothesis since no difference was found between two personality types. However, the third hypothesis cannot be rejected due to the correlation of Extraversion with TBR and DBR in many areas. Positive relationships between Extraversion and TBR in frontal lobe allows if extraverts have a lower attention control and more prone to disadvantageous decisions comparing to introverts. Positive association of Extraversion with DBR in parietal lobes predicts higher self-reported attention control. Since both central parietal and central frontal areas have differences in TBR, the difference in DMN functioning can exist. Positive correlation of Extraversion with most brain areas' TBR indicates that extraverts have higher domination of subcortical structures over the cortex. Association of DBR in the parietal lobe and left hemisphere with Extraversion can be explained by DBR relationship to testosterone/cortisol ratio [20]. However, this assumption results in a higher stress level in extraverts comparing to introverts, while previous research relates stress level to Neuroticism that did not have a correlation with DBR [21,22].

\section{Conclusion}

It is difficult to evaluate the existence of two groups with opposite Extraversion and Neuroticism measures in the general population due to the absence of normal distribution within the sample. Although extraversion related between-group difference in P4 TBR cannot be stated, correlation analysis supported its existence. There are relationships between extraversion and attention control. There are significant but small differences in brain functioning related to Extraversion that can be described as more dominant subcortical structures in extraverts comparing to introverts.

\section{Competing Interests}

The authors declare that they have no competing interests.

\section{References}

1. Derakshan N, Eysenck MW (2009) Anxiety, Processing Efficiency, and Cognitive Performance. European Psychologist 14(2): 168-176.

2. Knyazev GG (2007) Motivation, emotion, and their inhibitory control mirrored in brain oscillations. Neuroscience \& Biobehavioral Reviews 31: 377-395.

3. Schutter D J L G, Van Honk J (2005) Electrophysiological ratio markers for the balance between reward and punishment. Cognitive Brain Research 24: 685-690.
4. DeYoung CG (2010) Personality Neuroscience and the Biology of Traits. Social and Personality Psychology Compass 4(12): 1165-1180.

5. Gray JA (1973) Causal theories of personality and how to test them. In: J R Royce (Ed.), Multivariate analysis and psychological theory, Academic Press, Oxford, UK pp. 409-463.

6. Deckersbach T, Miller KK, Klibanski A, Fischman A, Dougherty D D, et al. (2006) Regional cerebral brain metabolism correlates of Neuroticism and Extraversion. Depress Anxiety 23(3): 133-138.

7. Netter P (2004) Personality and hormones. In: R M Stelmack (Ed.), On the Psychobiology of personality: Essays in Honor of Marvin Zuckerman, Elsevier, New York, US, pp. 353-377.

8. Putman P, van Peer J, Maimari I, van der Werff S (2010) EEG theta/beta ratio in relation to fear-modulated response-inhibition, attentional control, and affective traits. Biological Psychology 83: 73-78.

9. Arns M, Conners CK, Kraemer HC (2013) A decade of EEG theta/ beta ratio research in ADHD: A meta-analysis. Journal of Attention Disorders 17(5): 374-383.

10. Morillas-Romero A, Tortella-Feliu M, Bornas X, Putman $P$ (2015) Spontaneous EEG theta/beta ratio and delta-beta coupling in relation to attentional network functioning and self-reported attentional control. Cogn Affect Behav Neurosci 15(3): 598-606.

11. Paulus D J, Vanwoerden S, Norton P J, Sharp C (2016) From neuroticism to anxiety: Examining unique contributions of three transdiagnostic vulnerability factors. Personality and Individual Differences 94: 38-43.

12. Knyazev G, Merkulova E, Savostyanov A, Bocharov A, Saprigyn A (2019) Personality and EEG correlates of reactive social behavior. Neuropsychologia 124: 98-107.

13. Lei X, Yang T, Wu T (2015) Functional neuroimaging of extraversionintroversion. Neurosci Bull 31(6): 663-675.

14. Wacker J (2017) Effects of positive emotion, extraversion, and dopamine on cognitive stability-flexibility and frontal EEG asymmetry. Psychophysiology 55(1): e12727.

15. Schneider M, Chau L, Mohamadpour M, Stephens N, Arya K, et al. (2016) EEG asymmetry and BIS/BAS among healthy adolescents. Biol Psychol 120: 142-148.

16. Korjus K, Uusberg A, Uusberg H, Kuldkepp N, Kreegipuu K, et al. (2015) Personality cannot be predicted from the power of resting state EEG. Front Hum Neurosci 9: 63.

17. Rojas G M, Alvarez C, Montoya C E, de la Iglesia-Vayá M, Cisternas J E, et al. (2018) Study of resting-state functional connectivity networks using EEG electrodes position as seed. Front Neurosci 12.

18. Eysenck HJ (1968) Manual for the Eysenck Personality Inventory. In: Educational and Industrial Testing Service, San Diego, US.

19. Raygorodsky D Y (2001) Practical psychodiagnostics. Methods and tests. Tutorial. Publishing House" BAKHRAM-M: Samara, Russia.

20. Sroykham W, Wongsawat Y (2018) Estimation of testosterone/ cortisol ratio by resting state EEG delta/beta ratio in elderly people. Neuroendocrinol Lett 39(1): 75-82.

21. Gray JA, McNaughton N (2000) The Neuropsychology of Anxiety. In: An Enquiry into the Functions of the SeptoHippocampal System (Second Edition), Oxford University Press, New York, US.

22. Van Son D, Schalbroeck R, Angelidis A, van der Wee NJA, van der Does W, et al. (2018) Acute effects of caffeine on threat-selective attention: moderation by anxiety and EEG theta/beta ratio. Biol Psychol 136: 100-110. 
This work is licensed under Creative Commons Attribution 4.0 License DOI: 10.19080/PBSIJ.2019.11.555814

\section{Your next submission with Juniper Publishers} will reach you the below assets

- Quality Editorial service

- Swift Peer Review

- Reprints availability

- E-prints Service

- Manuscript Podcast for convenient understanding

- Global attainment for your research

- Manuscript accessibility in different formats ( Pdf, E-pub, Full Text, Audio)

- Unceasing customer service

Track the below URL for one-step submission https://juniperpublishers.com/online-submission.php 